\title{
Evolution of the Cosmological UV Background
}

\author{
Francesco Haardt* \\ Dipartimento di Fisica \& Matematica, Università dell'Insubria, Como, Italy \\ Department of Astronomy \& Astrophysics, University of California, Santa Cruz (CA), USA \\ E-mail: haardt@uninsubria.it
}

\section{Piero Madau}

Department of Astronomy \& Astrophysics, University of California, Santa Cruz (CA), USA

E-mail: pmadau@ucolick.org

\begin{abstract}
We present improved synthesis models of the evolving spectrum of the UV/X-ray diffuse background, updating and extending our previous results. A "minimal cosmic reionization model" is presented in which the galaxy UV emissivity traces recent determinations of the cosmic history of star formation, the luminosity-weighted escape fraction of hydrogen-ionizing radiation increases rapidly with redshift, from $1 \%$ at $z=3$ to $50 \%$ at $z=8.5$, the clumping factor of the intergalactic medium is $C_{\mathrm{IGM}}=3$, and Population III stars make a negligible contribution to the metagalactic flux. The model provides a good fit to the hydrogen-ionization rates inferred from flux decrement measurements, predicts that cosmological HII regions overlap at redshift 7.5, and yields an optical depth to Thomson scattering, $\tau_{e}=0.085$, that is agreement with WMAP results.
\end{abstract}

25th Texas Symposium on Relativistic Astrophysics - TEXAS 2010

December 06-10, 2010

Heidelberg, Germany

*Speaker. 


\section{Introduction}

The reionization of the all-pervading intergalactic medium (IGM) is a landmark event in the history of cosmological structure formation. Studies of Gunn-Peterson absorption in the spectra of distant quasars show that hydrogen is highly photoionized out to redshift $z>6$, while polarization data from the Wilkinson Microwave Anisotropy Probe (WMAP) constrain the redshift of a sudden reionization event to be significantly higher, $z=11.0 \pm 1.4$. It is generally thought that the IGM is kept ionized by the integrated UV emission from active nuclei and star-forming galaxies, but the relative contributions of these sources as a function of epoch are poorly known. In this contribution we describe a new version of CUBA and use it to compute improved synthesis models of the UV/Xray cosmic background spectrum and evolution, combining, updating, and extending many of our previous results in this field. Thorough details and all relevant references can be found in Haardt \& Madau (2011).

\section{Cosmological radiative transfer}

The equation of cosmological radiative transfer describing the time evolution of the space- and angle-averaged monochromatic intensity $J_{v}$ is

$$
\left(\frac{\partial}{\partial t}-v H \frac{\partial}{\partial v}\right) J_{v}+3 H J_{v}=-c \kappa_{v} J_{v}+\frac{c}{4 \pi} \varepsilon_{v}
$$

where $H(z)$ is the Hubble parameter, $c$ the speed of the light, $\kappa_{v}$ is the absorption coefficient, and $\varepsilon_{v}$ the proper volume emissivity. The integration of equation (2.1) gives the background intensity at the observed frequency $v_{o}$, as seen by an observer at redshift $z_{o}$,

$$
J_{v_{o}}\left(z_{o}\right)=\frac{c}{4 \pi} \int_{z_{o}}^{\infty}|d t / d z| d z \frac{\left(1+z_{o}\right)^{3}}{(1+z)^{3}} \varepsilon_{v}(z) e^{-\bar{\tau}}
$$

where $v=v_{o}(1+z) /\left(1+z_{o}\right),|d t / d z|=H^{-1}(1+z)^{-1}, \bar{\tau} \equiv-\ln \left\langle e^{-\tau}\right\rangle$ is the effective absorption optical depth of a clumpy IGM, and $\varepsilon_{v}$ is the proper volume emissivity.

\subsection{Absorption}

The effective opacity of the IGM has traditionally been one of the main uncertainties affecting calculations of the UV background. Our improved model uses a piecewise power-law parameterization for the distribution of absorbers along the line of sight,

$$
f\left(N_{\mathrm{HI}}, z\right)=A N_{\mathrm{HI}}^{-\beta}(1+z)^{\gamma},
$$

and is designed to reproduce accurately a number of recent observations.

- Over the column density range $10^{11}<N_{\mathrm{HI}}<10^{15} \mathrm{~cm}^{-2}$, we use $(A, \beta, \gamma)=\left(1.2 \times 10^{7}, 1.5,3.0\right)$, where the normalization $A$ is expressed in units of $\mathrm{cm}^{-2(\beta-1)}$, and $\beta=1.5$.

- At the other end of the column density distribution, with a power-law exponent $\beta=2$ down to a break column of $N_{\mathrm{HI}}=10^{21.55} \mathrm{~cm}^{-2}$, and with an incidence per unit redshift $\propto(1+z)^{1.27}$, the parameters for the DLAs becomes $(A, \beta, \gamma)=\left(8.7 \times 10^{18}, 2,1.27\right)$. 
- For absorbers with $10^{19}<N_{\mathrm{HI}}<10^{20.3} \mathrm{~cm}^{-2}$ (the so-called "super Lyman-limit systems", or SLLSs), we use $(A, \beta, \gamma)=(0.45,1.05,1.27)$.

- There is obviously a significant mismatch between the power-law exponent for the Ly $\alpha$ clouds $(\gamma=3)$ and the SLLSs $(\gamma=1.27)$. Continuity then requires the shape of $f\left(N_{\mathrm{HI}}, z\right)$ to change with redshift over the colum density range of the Lyman-limit systems (LLSs), $10^{17.5}<N_{\mathrm{HI}}<10^{19} \mathrm{~cm}^{-2}$. In this interval of column densities we match the distribution function with a power law of redshift-dependent slope. The procedure yields the slopes $\beta=0.47,0.61,0.72,0.82$ at redshifts $z=2,3,4,5$, respectively.

- The above parameterizations reproduce well the observations at $2<z<5$. At low redshift, however, $H S T$ data show that the forest undergoes a much slower evolution. We take $\gamma=0.16$ in the interval $0<z<z_{\text {low }}$ and $d N / d z=34.7$ at $z=0$ above an equivalent width of 0.24 ÅWe derive $(A, \beta, \gamma)=\left(1.73 \times 10^{8}, 1.5,0.16\right)$ for $10^{11}<N_{\mathrm{HI}}<10^{15} \mathrm{~cm}^{-2}$ and $(A, \beta, \gamma)=$ $\left(5.49 \times 10^{15}, 2,0.16\right)$ for $10^{15}<N_{\mathrm{HI}}<10^{17.5} \mathrm{~cm}^{-2}$ at all redshifts below $z_{\text {low }}=1.56$. We use a broken power-law for the redshift distribution of the SLLSs and DLAs as well; assuming that the same $\gamma=0.16$ slope and transition redshift $z_{\text {low }}$ inferred for the forest also hold in the case of the thicker absorbers, we derive a normalization at $z<z_{\text {low }}$ of $A=1.28$ for the SLLSs and $A=2.47 \times 10^{19}$ for the DLAs. This yields $d N / d z=0.74$ absorbers above $N_{\mathrm{HI}}=10^{17.2} \mathrm{~cm}^{-2}$ at $\langle z\rangle=0.69$.

- Above $z=5.5$ we assume for the forest the values $(A, \beta, \gamma)=(29.5,1.5,9.9)\left(10^{11}<N_{\mathrm{HI}}<\right.$ $\left.10^{15} \mathrm{~cm}^{-2}\right)$ and $(A, \beta, \gamma)=\left(9.35 \times 10^{8}, 2,9.9\right)\left(10^{15}<N_{\mathrm{HI}}<10^{17.5} \mathrm{~cm}^{-2}\right)$ above redshift 5.5 .

\subsection{Emission}

The emissivity is due to several contributing terms:

- The background photons absorbed through a Lyman series resonance cause a radiative cascade that ultimately terminates either in a Ly $\alpha$ photon or in two-photon $2 s \rightarrow 1 s$ continuum decay. We used the detailed photoionization structure of absorbing systems to calculate the reprocessing of background LyC radiation by the clumpy IGM via atomic recombination processes. We included recombinations from the continuum to the ground state of HI, HeI, and HeII, as well as HeII Balmer, two-photon, and Ly $\alpha$ emission.

- The adopted quasar comoving emissivity at $1 \mathrm{Ryd}, \varepsilon_{912}(z) /(1+z)^{3}$,

$$
\frac{\varepsilon_{912}(z)}{(1+z)^{3}}=\left(10^{24.6} \operatorname{ergs~Mpc}^{-3} \mathrm{~s}^{-1} \mathrm{~Hz}^{-1}\right)(1+z)^{4.68} \frac{\exp (-0.28 z)}{\exp (1.77 z)+26.3}
$$

which closely fits the observational results in the range $1<z<5.7$ under the assumption of pure luminosity evolution. The poorly known faint-end slope of the quasar luminosity function at high redshift, incompleteness corrections, as well as the uncertain spectral energy distribution (SED) in the UV, all contribute to the large apparent discrepancies between different authors. In the following we shall use the functional form given above together with the broken power-law SED with $L_{v} \propto v^{-0.44}$ for $1300<\lambda<5000$, and $L_{v} \propto v^{-1.57}$ for $\lambda<1300$. 
- Star-forming galaxies are expected to play a dominant role as sources of hydrogen-ionizing radiation at $z>3$ as the quasar population declines with lookback time. To compute the LyC emissivity from galaxies at all epochs, we start with an empirical determination of the star formation history of the universe. We adopt the far-UV (FUV, $1500 \AA$ ) published luminosity functions in the redshift range $0<z<9$, integrated down to $L_{\min }=0.01 L_{*}$ using Schechter function fits with parameters $\left(\phi_{*}, L_{*}, \alpha\right)$ to compute the dust-reddened galaxy FUV luminosity density $\rho_{\text {FUV }}$. Dust attenuation was treated using a Calzetti extinction law normalized at $1500 \AA$ A . Finally, the dust-corrected luminosity densities were smoothed with an approximating function and then compared with the results of spectral population synthesis models provided by the GALEXEV library.

\section{Background Synthesis}

Figure 1 shows the quasar-only background spectrum generated by an upgraded version of our radiative transfer code CUBA, using the formalism and parameters described above. CUBA solves the radiative transfer equation by iteration, as its right-hand term implicitly contains $J$ in the recombination emissivity and in the effective helium opacity. Physically, this simply means that the metagalactic UV flux depends on the ionization state of intervening absorbers, which is in turn determined by background radiation. For comparison, we have also plotted the background spectrum from our old models. The new models are characterized by a lower UV flux (by as much as a factor of 3 at $1 \mathrm{Ryd}$ and $z=3$ ), smaller spectral breaks from HI and HeII LyC absorption, a sawtooth modulation by the Lyman series of HI and HeII that becomes more and more substantial with redshift.

Figure 2 shows the full background (i.e., quasar plus galaxies) compared to the quasar-only spectrum. The much softer emissivity, linked to HI absorption fixed at the observed level, causes a much deeper HeII absorption trough, and much deeper HeII Ly series absorption lines.

Figure 3 shows our predicted HI ionization rate compared to several measurements. The full model provides a good fit to the hydrogen-ionization rates inferred from flux decrement measurements, predicts that cosmological HII regions overlap at redshift 7.5, and yields an optical depth to Thomson scattering, $\tau_{e}=0.085$, that is in agreement with WMAP results.

\section{References}

[1] F. Haardt F. \& P. Madau, P., Radiative Transfer in a Clumpy Univers. IV. New Synthesis Models of the Cosmic UV/X-Ray Backround, ApJ, 2011, to be submitted

[2] F. Haardt \& P. Madau, Radiative Transfer in a Clumpy Universe. II. The Ultraviolet Extragalactic Background, ApJ, 1996, 461, 20 


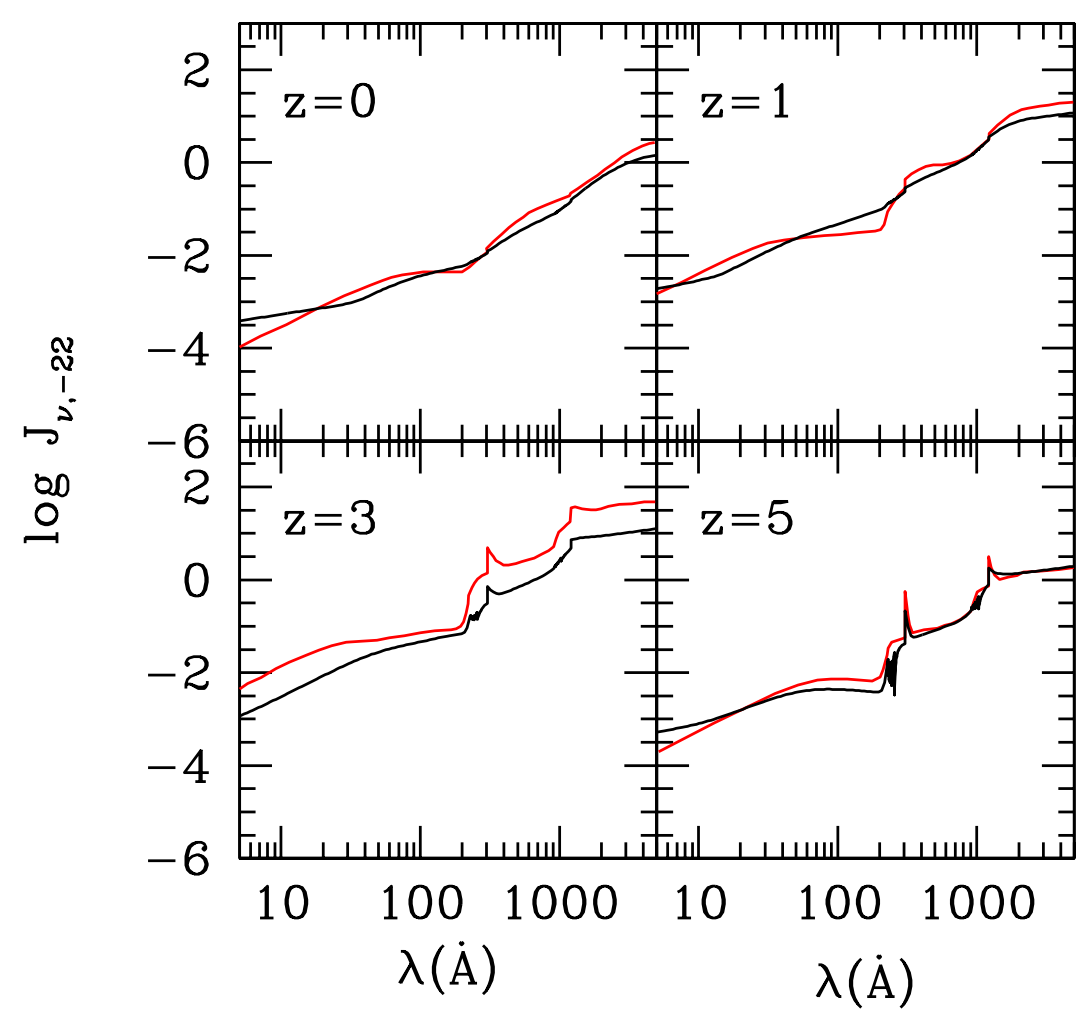

Figure 1: The cosmic background spectrum from quasars only between $5 \AA$ and $5,000 \AA$ at epochs $z=0,1,3$,and 5. The new models (black curves) are compared with the old results of Haardt \& Madau (1996)(red curves). The intensity $J_{v}$ is expressed in units of $10^{-22} \mathrm{ergs} \mathrm{cm}^{-2} \mathrm{~s}^{-1} \mathrm{~Hz}^{-1} \mathrm{sr}^{-1}$. 


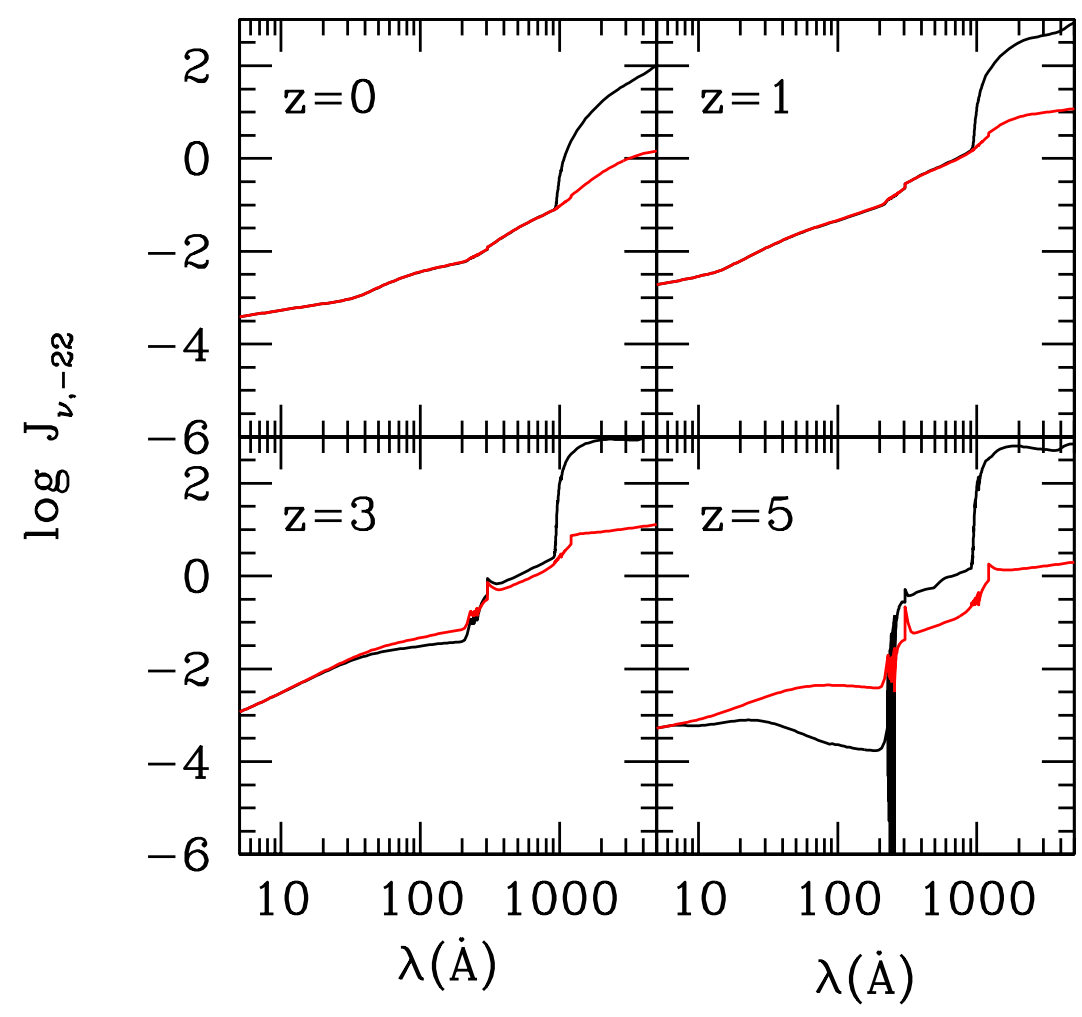

Figure 2: Same as above, but now the red line is the new quasar-only background, compared to the full (quasars plus galaxies) model black line). 


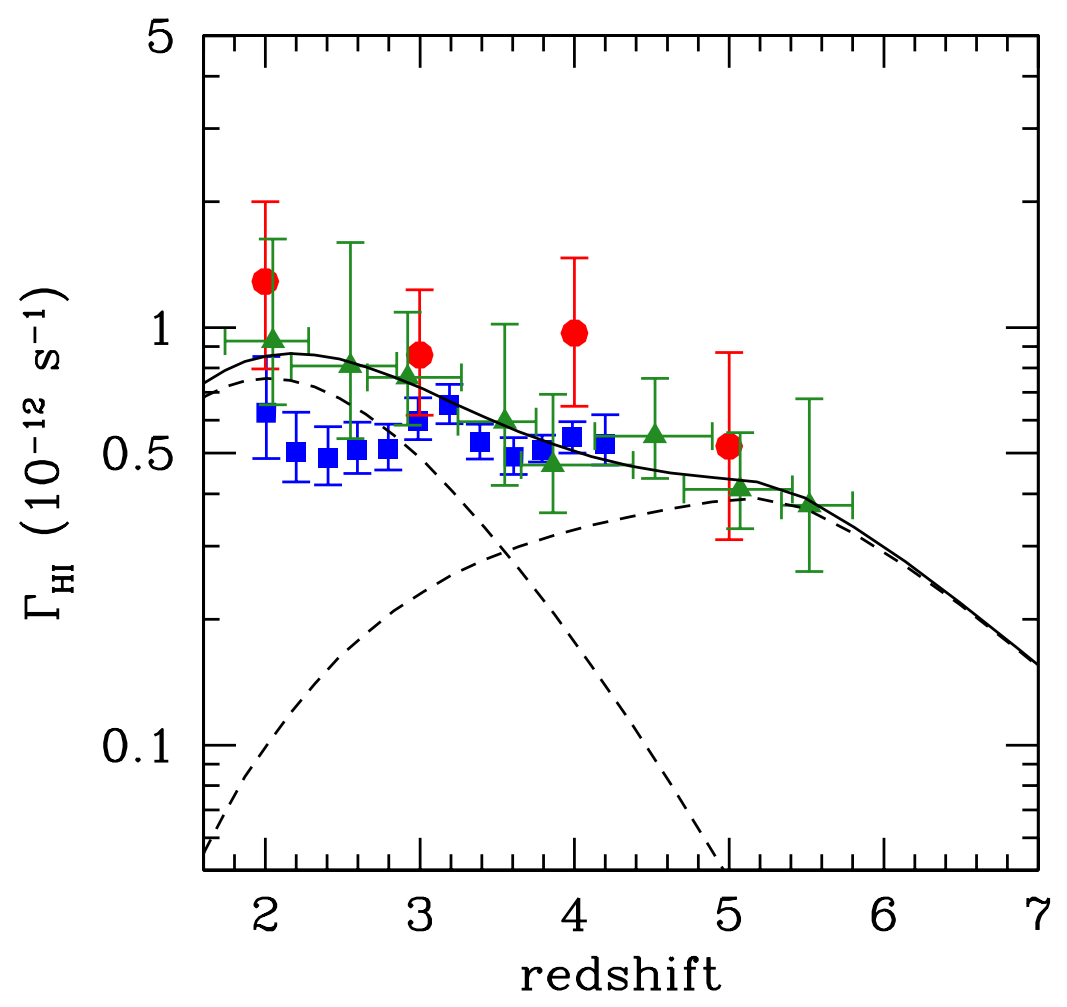

Figure 3: Hydrogen photoionization rate as a function of redshift. Solid curve: our full model. Dashed curves: separate contributions from quasars (leftmost curve) and galaxies (rightmost curve). Different data points refer to various empirical measurements from the Ly $\alpha$ forest effective opacity (see Haardt \& Madau 2011 for full references). 